\title{
Correction to: Downregulation of GLYR1 contributes to microsatellite instability colorectal cancer by targeting p21 via the p38MAPK and PI3K/AKT pathways
}

Zhiyan $\mathrm{Hu}^{2,3,4+}$, Ting Long ${ }^{3,4+}$, Yidan Ma,4, Jiaxian Zhu ${ }^{3,4}$, Lingfang Gao ${ }^{3,4}$, Yan Zhong ${ }^{3,4}$, Xia Wang ${ }^{2,3,4}$, Xiaoyan Wang ${ }^{2,3,4}$ and Zuguo Li $\mathrm{il}^{1,3,4^{*}}$

\section{Correction to: J Exp Clin Cancer Res 39, 76 (2020) https://doi.org/10.1186/s13046-020-01578-y}

Following publication of the original article [1], the authors identified an error in the author author's affiliation.

The incorrect author's affiliation is:

Zhiyan $\mathrm{Hu}^{1,2,3 \dagger}$, Ting Long ${ }^{2,3+}$, Yidan $\mathrm{Ma}^{2,3}$, Jiaxian $\mathrm{Zhu}^{2,3}$, Lingfang Gao ${ }^{2,3}$, Yan Zhong ${ }^{2,3}$, Xia Wang ${ }^{1,2,3}$, Xiaoyan Wang ${ }^{1,2,3}$, Zuguo Li ${ }^{4,5,6^{*}}$

1 Department of Pathology, Shenzhen Hospital of Southern Medical University, Shenzhen, China.

${ }^{2}$ Department of Pathology, Nanfang Hospital, Southern Medical University, Guangzhou, China.

${ }^{3}$ Department of Pathology, School of Basic Medical Sciences, Southern Medical University, Guangzhou, China.

${ }^{4}$ Guangdong Provincial Key Laboratory of Molecular tumor Pathology, Guangzhou, China.

${ }^{5}$ Department of Pathology, School of Basic Medical Sciences, Southern Medical University,

${ }^{6}$ Guangdong Provincial Key Laboratory of Molecular tumor Pathology, Guangzhou, China.

\section{The original article can be found online at https://doi.org/10.1186/s13046} 020-01578-y

* Correspondence: Lizg@smu.edu.cn

${ }^{\dagger}$ Zhiyan Hu and Ting Long contributed equally to this work.

'Department of Pathology, Shenzhen Hospital of Southern Medical University, Shenzhen, China

${ }^{3}$ Department of Pathology, School of Basic Medical Sciences, Southern Medical University, Guangzhou, China

Full list of author information is available at the end of the article
The correct author's affiliation is:

Zhiyan $\mathrm{Hu}^{2,3,4 \dagger}$, Ting Long ${ }^{3,4 \dagger}$, Yidan $\mathrm{Ma}^{3,4}$, Jiaxian Zhu $^{3,4}$, Lingfang Gao ${ }^{3,4}$, Yan Zhong ${ }^{3,4}$, Xia Wang ${ }^{2,3,4}$, Xiaoyan Wang ${ }^{2,3,4}$ and Zuguo $\mathrm{Li}^{1,3,4^{*}}$

1 Department of Pathology, Shenzhen Hospital of Southern Medical University, Shenzhen, China.

${ }^{2}$ Department of Pathology, Nanfang Hospital, Southern Medical University, Guangzhou, China.

${ }^{3}$ Department of Pathology, School of Basic Medical Sciences, Southern Medical University, Guangzhou, China.

${ }^{4}$ Guangdong Provincial Key Laboratory of Molecular tumor Pathology, Guangzhou, China.

The author group has been updated above and the original article [1] has been corrected.

\section{Author details}

'Department of Pathology, Shenzhen Hospital of Southern Medical University, Shenzhen, China. '2Department of Pathology, Nanfang Hospital, Southern Medical University, Guangzhou, China. ${ }^{3}$ Department of Pathology, School of Basic Medical Sciences, Southern Medical University, Guangzhou, China. ${ }^{4}$ Guangdong Provincial Key Laboratory of Molecular tumor Pathology, Guangzhou, China.

Published online: 06 July 2020

Reference

1. Hu, et al. Downregulation of GLYR1 contributes to microsatellite instability colorectal cancer by targeting p21 via the p38MAPK and PI3KAKT pathways. J Exp Clin Cancer Res. 2020;39:76. 